\title{
Formulación de una solución antifúngica para la Preservación del Archivo Histórico de la Universidad Central del Ecuador
}

\author{
MARCELO D. CAMPUÉS ORDOÑEZ', LILIANA NARANJO', JAVIER RODRIGO SANTAMARÍA-AGUIRRE ${ }^{*}$ \\ NATASHA SANMARTÍN'², TROSKY YANEZ', PABLO BONILLA' \\ 'Facultad de Ciencias Químicas, Universidad Central del Ecuador, Quito., \\ ${ }^{2}$ Área Histórica, Archivo Histórico, Universidad Central del Ecuador, Quito. \\ *Correspondencia: jrsantamaria@uce.edu.ec
}

Recibido: 2 octubre 2016, Aceptado: 30 agosto 2017

\section{Resumen}

La conservación documental en los últimos años cumple un rol importante en la sociedad, debido a que trata de recuperar la memoria histórica y el acervo documental de la misma. Uno de los problemas de mayor incidencia y dificultad es la presencia de hongos y sus productos metabólicos en los documentos, ya que potencialmente ocasionan la pérdida total de la información dificultando de esta forma los procesos de restauración. El presente estudio busca formular una solución para tratar esta contaminación de hongos, con el uso de fluconazol como agente antifúngico. Se inició aislando los hongos presentes en los libros, seguido del uso del Diagrama pseudoternario para la formulación de la solución de Fluconazol, y por último, se evaluó la eficacia de esta formulación por técnicas microbiológicas modificadas, obteniéndose de esta manera, una reducción porcentual de Unidades Formadoras de Colonias de hongos mayor al 90\%, mediante la nebulización de una solución de Fluconazol al $24 \%$ compuesta por una mezcla de cosolventes: Agua: 20\%, Metanol: 50\%, Etanol: 30\%, con un tiempo de aplicación de 15 minutos.

Palabras clave: Conservación documental, Fluconazol, Solución antifúngica, Nebulización.

\section{Formulation of an antifungal solution for the preservation of the historical archive of the Central Ecuador University}

\section{Abstract}

The documentary conservation in recent years plays an important role in society, because it tries to recover the historical memory, and the documentary acquis of it. One of the most prevalent and difficult problems is the existence of fungi and its metabolic products in documents, as it potentially causes a total loss of information, hindering the restoring process as well. The study seeks to formulate a solution to treat this fungal contamination, using Fluconazole as an antifungal. We started by isolating fungi found in the books, followed by the use of the pseudo-ternary diagram for the formulation of the solution of Fluconazole, and finally the effectiveness of this formulations was evaluated by using modified microbiological techniques, obtaining a percentage reduction on CFU of $90 \%$, by nebulization a solution of Fluconazole at $24 \%$ and co-solvents: Water: $20 \%$, Methanol: 50\%, Ethanol: 30\%, during 15 minutes.

Keywords: Documentary conservation, Fluconazole, Antifungal solution, Nebulization. 


\section{Introducción}

Los Centros documentarios o reservas históricas vienen cumpliendo un papel fundamental dentro de la sociedad, pues aportan a la comunidad mediante, la gestión de acciones, la educación, el desarrollo cultural, la toma de decisiones, la transparencia en las acciones administrativas, los avances en la educación, la cultura e investigación. A ellos se han venido vinculando profesiones de diferentes campos aportando a la conservación y preservación de los centros documentarios.

En la actualidad se da importancia al rescate de los documentos bibliográficos de las áreas Históricas, y son varios proyectos de conservación documental, que se han visto limitados al tratamiento tradicional de los documentos físicos, sin promover nuevos estudios científicos en este campo. En este contexto es que se han detectado varios problemas, siendo uno de los más importantes los agentes fúngicos que afectan a las fuentes bibliográficas físicas y sus espacios. [1]

El Área Histórica de la Universidad Central del Ecuador cuenta con un acervo documental inmenso, contiene alrededor de 30.000 libros y documentos del fondo antiguo de la Universidad que están comprendidos entre los años 1485 y 1960, abarcado un período de cinco siglos.

Entre los tratamientos tradicionales para la restauración de libros se encuentra el uso de ciertas técnicas para la eliminación de hongos siendo las más predominantes: el uso de atmosferas de dióxido de carbono y el tratamiento por congelación al vacío.

Los hongos son organismos eucariotas típicos que contribuyen a la descomposición de la materia orgánica, participando en los distintos ciclos biológicos. Los hongos presentes en documentos bibliográficos se los encuentra en forma de esporas y eucariotas desarrollados. El tipo y la extensión de estos contaminantes fúngicos depende de las características del libro como de las condiciones ambientales a las que se encuentre el mismo. Entre las condiciones ambientales óptimas para el crecimiento se encuentran: una alta humedad (40\%), temperatura ambiental $\left(21^{\circ} \mathrm{C}\right)$, poca cantidad de luz, y baja circulación de aire.[2]

Los antimicóticos son fármacos que tienen la capacidad de evitar el crecimiento de hongos e incluso provocar la muerte de los mismos, por lo que se usan para tratar distintas enfermedades en humanos. El Fluconazol es un agente antimicótico de amplio es- pectro, comúnmente usado en tratamientos debido a sus excelentes propiedades fisicoquímicas y su escasa toxicidad.[3].A la fecha no se han encontrado estudios de Fluconazol en superficies inertes.

Una herramienta utilizada para formular soluciones de fármacos por cosolvencia es el diagrama pseudoternario en el que cada uno de los componentes puros están representados en cada vértice y pueden ser expresados mediante \% peso/peso o Molaridad. La suma de los tres componentes debe llegar a 100\%.[4]

El objetivo principal de esta investigación fue formular una solución anti fúngica capaz de eliminar los hongos presentes en los libros sin alterar sus características físicas, para lo cual en primera instancia se aislaron e identificaron los hongos presentes en los libros del Archivo Histórico de la Universidad Central de Ecuador, para luego formular una solución anti fúngica usando Fluconazol, y al final evaluar la eficacia de estas soluciones formuladas.

\section{Parte Experimental}

Equipos y Materiales: Para el aislamiento de los hongos se utilizó como medio de cultivo Sabouraud Dextrosa Agar, para la identificación de los mismos se observó su estructura y morfología con el uso de un microscopio (Lumin) y las técnicas de tinción Gram para bacterias y el reactivo de Azul de Lactofenol para hongos . La incubadora que se utilizo fue Gemmyco Digital \# IN-601. Durante la elaboración de las Formulaciones se utilizó como materia prima Fluconazol, Etanol (96\%), Metanol técnico (USP), Agua destilada tipo 2. En la valoración del Fluconazol se utilizó un espectrofotómetro UV -VIS $(\mathrm{HACH})$. Para la esterilización de todo el material se usó un autoclave vertical (BIOBASE), y se trabajó todo el procedimiento microbiológico en un cabina de flujo laminar (Flow 85V). Para la aplicación de las soluciones se utilizó un nebulizador (TFO1), con capacidad de 2,2 L y tasa de evaporación 250 mililitros por hora.

\section{Métodos de valoración de la materia prima} Fluconazol: Se utilizó el método espectrofotométrico descrito en la Farmacopea Brasileña 2010 [5], la cual brevemente describe que se debe pesar un equivalente a $100 \mathrm{mg}$ de Fluconazol en un matraz $100 \mathrm{~mL}$. Añadir $70 \mathrm{~mL}$ de ácido clorhídrico $0,1 \mathrm{M}$, dejar en ultrasonido durante 10 minutos, aforar con el mismo disolvente, homogenizar y filtrar. Tomar $10 \mathrm{~mL}$ de esta solución y diluir con ácido clorhídrico $0,1 \mathrm{M}$ en un matraz aforado de $50 \mathrm{~mL}$ hasta alcanzar una concentración de $0,02 \%(\mathrm{p} / \mathrm{v})$. Leer en el espectrofotómetro a $261 \mathrm{~nm}$. Este procedimiento se realiza para la muestra y estándar. 
Análisis microbiológico del aire por sedimentación en placa: Este método se aplica en las áreas de almacenamiento del Área Histórica, donde en primer lugar se evalúa las áreas con mayor riesgo de contaminación a ser evaluadas, luego con las cajas Petri destapadas se colocan boca arriba y se las deja por 3 horas, al cabo de este tiempo se cierran las cajas y se incuban durante 7 días a $37^{\circ} \mathrm{C}$. Se cuentan las colonias formadas y se reportan los datos respectivos[6].

Análisis de superficies no planas (libros) por hisopado: Se utilizó la técnica modificada descrita en [6], donde se coloca una plantilla $5 \times 5$ sobre la superficie a ser muestreada, con la ayuda de un hisopo estéril se fricciona sobre la superficie del libro en todas las direcciones, se coloca dentro de un tubo estéril que contiene $10 \mathrm{ml}$ se solución salina y se agita enérgicamente por 1 minuto y luego se lo deja reposar 15 minutos, se siembra por extensión $0,1 \mathrm{ml}$ de la solución en cajas Petri, se incuba a $37^{\circ} \mathrm{C}$ por 7 días y se cuenta las colonias formadas.

Identificación de hongos por la prueba de azul de Lactofenol: Se toma una fracción de las colonias aisladas a ser identificadas, con la ayuda de una asa microbiológica y cinta adhesiva de $1 \mathrm{~cm} \times 1 \mathrm{~cm}$ pegada al extremo del asa, con la superficie adhesiva tocar la parte superior de la colonia, colocar una gota de azul de Lactofenol en el porta objetos y pegar la cinta adhesiva sobre él, colocar una nueva gota sobre la cinta y cubrir con el cubreobjetos, Observar a 40x en el microscopio.[6]

Preparaciones de las soluciones antifungicas: Para la preparación de cada solución, se pesa la cantidad de Fluconazol correspondiente a la proporción del diagrama pseudeternario, se miden los volúmenes de etanol, metanol y agua de acuerdo al mismo diagrama, con la ayuda de un agitador magnético mezclar el metanol, etanol y agua con agitación constante y a temperatura ambiente, luego con la ayuda de una espátula colocar poco a poco el Fluconazol en la mezcla hasta observar una completa disolución.

\section{Análisis de la eficacia antifúngica de las so-} luciones de Fluconazol: Esta técnica se realizó mediante el método de muestreo en superficies irregulares con hisopo descrito anteriormente, antes y después de la nebulización de las distintas soluciones. Para lo cual de los libros presuntamente contaminados con hongos: 190 se tomó una muestra representativa con la Tabla Militarizada Estándar en un nivel de inspección N-2 obteniéndose 32 libros elegidos completamente al azar. A continuación sobre las hojas de un libro elegidas aleatoriamente se realiza el muestreo, se aplica la nebulización por el tiempo estimado en el diseño experimental y luego se realiza el muestreó final inmediatamente concluida la nebulización en las mismas hojas, el ensayo se realiza por triplicado, en tres hojas diferentes del mismo libro.

Diseño experimental: El diseño experimental se realizó para la eficacia anti fúngica y consiste en un Diseño Factorial Multivariable Completamente al Azar, con 2 Factores: Soluciones de Fluconazol, con 3 niveles y Tiempo de Aplicación, con dos niveles; con 3 repeticiones por cada ensayo siendo un total de 18 tratamientos realizados, midiendo como variable respuesta la reducción porcentual de UFC de hongos, analizados mediante un análisis de varianza y Optimización de Respuesta.

\section{Resultados y Discusión}

\section{Microorganismos aislados por muestreo en el aire}

Tabla 1. Microorganismos aislados por muestreo de aire.

\begin{tabular}{ccc}
\hline Área de Riesgo & $\begin{array}{c}\text { Promedio de Unidades } \\
\text { Formadoras de colonias } \\
\text { por caja (UFC/caja) }\end{array}$ & Identificación \\
\hline Área Cuarentena & 25 & Aspergillus sp, Penicillium sp. \\
Pasillo General del Área Histórica & 2 & Aspergillus sp. \\
Sala de Extracción de Aire & 15 & Aspergillus sp, Penicilliumsp. \\
Archivo Histórico & 6 & Aspergillus sp,Penicillium sp.
\end{tabular}


Como se puede observar en la Tabla 1 las familias más predominantes en el aire de la instalación del Área Histórica son dos: Aspergillus sp y Penicillium sp. Se observa también que las áreas más contaminadas son La sala de Cuarentena y la de Extracción de Aire, por lo que los libros ubicados en estos sitios son lo más contaminados.

\section{Microorganismos aislados por muestreo en libros contaminados}

Tabla 2. Microorganismos aislados por muestreo en libros

\begin{tabular}{|c|c|c|c|}
\hline Numero de Libros & Año de Publicación & $\begin{array}{c}\text { Promedio de Unidades } \\
\text { Formadoras de Colonias } \\
\text { UFC } / \mathrm{cm}^{2}\end{array}$ & Identificación \\
\hline 5 & 1500 & 25 & Aspergillus sp, Penicilliumsp. \\
\hline 11 & 1600 & 64 & Aspergillus sp, Penicilliumsp. \\
\hline 6 & 1700 & 6 & Aspergillus sp, Penicilliumsp. \\
\hline 6 & 1800 & 12 & Aspergillus sp, Penicilliumsp. \\
\hline 4 & 1900 & 9 & Aspergillus sp, Penicilliumsp. \\
\hline
\end{tabular}

Como se puede observar en la Tabla 2 las familias más predominantes en las hojas de los libros son dos: Aspergillus sp, y Penicillium sp., coincidiendo con los aislados en el aire. El número de hongos aislados es mayor en los siglos 1500 y 1600 debido probablemente a la elevada cantidad de celulosa que se usaba para realizar libros en esa época. [7] Las características morfológicas y microscópicas de las dos familias obtenidas son:

Tabla 3. Caracterización de los hongos aislados.

\begin{tabular}{ccc}
\hline Familia & Descripción & Microscópica \\
\hline Penicillium & $\begin{array}{c}\text { Colonias aterciopeladas elevadas de } \\
\text { color verde-gris. } \\
\text { Colonias elevadas pulverulentas de } \\
\text { color blanco }\end{array}$ & $\begin{array}{c}\text { Conidióforos rectos y hialinos, Conidias } \\
\text { en cadena, esféricas y hialinas }\end{array}$ \\
Aspergillus & Colonias planas pulverulentas de \\
& color verde & $\begin{array}{c}\text { Conidioforos hialinos de pared rugosa, } \\
\text { Cabezuela radiada, biseriada, Conidias } \\
\text { equinuladas, esféricas }\end{array}$ \\
\hline
\end{tabular}

Las características descritas en la Tabla 3 coinciden con la bibliografía [8], tanto en observación macroscópica: la forma, el color; la apariencia de los hongos, como en la observación microscópica hifas, conidios, micelios.

\section{Formulación de la solución antifúngica}

Una vez que se identificaron las familias de hongos presentes en los libros se seleccionó al Fluconazol como mejor alternativa para el tratamiento de los 
mismos, debido a su alta solubilidad, menor grado tóxico y amplio espectro de acción[9]. La comparación de la gran mayoría de agentes anti fúngicos. La solución a ser el vehículo del Fluconazol debía cumplir ciertas condiciones como: no mojar excesivamente las hojas del libro, disolver la mayor cantidad posible de Fluconazol, y tener la capacidad de nebulizar, seleccionándose así como solventes: Etanol, Metanol y Agua [10]. La proporción adecuada de los solventes se determinó usando un diagrama pseudoternario, en donde se tomaron en una primera etapa 19 puntos y luego en una segunda etapa 10 puntos en la zona con mejores resultados obteniéndose así la Figura 1:

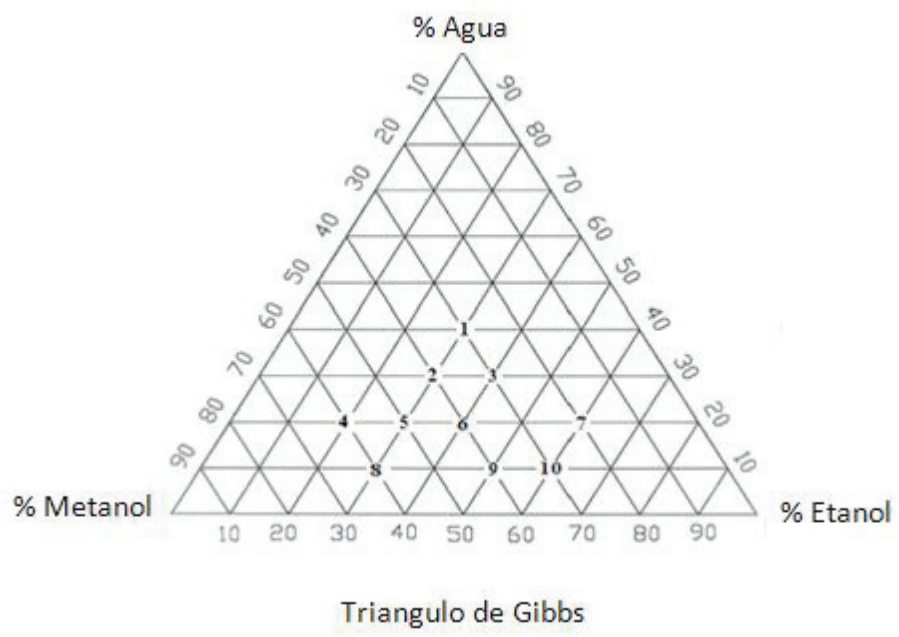

Figura 1. Diagrama pseudoternario con los 10 mejores resultados de \% solubilidad, nivel de nebulización y daño físico perceptible

Una vez que se determinó el porcentaje disuelto de Fluconazol de cada una de las combinaciones se obtuvo la siguiente Tabla 4:

Tabla 4. Elección de las proporciones adecuadas de los solventes.

\begin{tabular}{|c|c|c|c|c|c|c|}
\hline $\mathrm{N}-$ & $\%$ Agua & $\%$ Etanol & $\%$ Metanol & $\begin{array}{l}\text { \% Fluconazol } \\
\text { disuelto }\end{array}$ & $\begin{array}{l}\text { Apreciación de las } \\
\text { goticulas de } \\
\text { Nebulización }\end{array}$ & $\begin{array}{l}\text { Apreciación de la } \\
\text { Humedad del libro }\end{array}$ \\
\hline 1 & 40 & 30 & 30 & 13 & Alta & Poca \\
\hline 2 & 30 & 30 & 40 & 16 & Alta & Poca \\
\hline 3 & 30 & 40 & 30 & 15 & Alta & Poca \\
\hline 4 & 20 & 20 & 60 & 22 & Media & Ninguna \\
\hline 5 & 20 & 30 & 50 & 24 & Media & Ninguna \\
\hline 6 & 20 & 40 & 40 & 23 & Media & Ninguna \\
\hline 7 & 20 & 60 & 20 & 20 & Media & Ninguna \\
\hline 8 & 10 & 30 & 60 & 24 & Baja & Ninguna \\
\hline 9 & 10 & 50 & 40 & 27 & Baja & Ninguna \\
\hline 10 & 10 & 60 & 30 & 30 & Baja & Ninguna \\
\hline
\end{tabular}


Como puede observarse en la Tabla 4, los puntos 5, 9 , y 10 son los que presentan mayor proporción de Fluconazol disuelto, una nebulización aceptable e imperceptible humedad sobre la hoja del libro (Figura 2). A las tres soluciones se evaluó la eficacia antifúngica.

\section{Evaluación de la eficacia de las soluciones antifúngicas}

Para cada tipo de formulación y a cada tiempo determinado de acuerdo al diseño, se evaluó la eficacia expresada como reducción porcentual mediante la siguiente fórmula:

$$
\begin{aligned}
\% \text { No Reducido } & =\frac{\frac{u f c}{c m^{2}} \text { final }}{\frac{u f c}{c m^{2}} \text { inicial }} \times 100 \% \\
\% \text { Reducido } & =100 \%-\% \text { No reducido }
\end{aligned}
$$

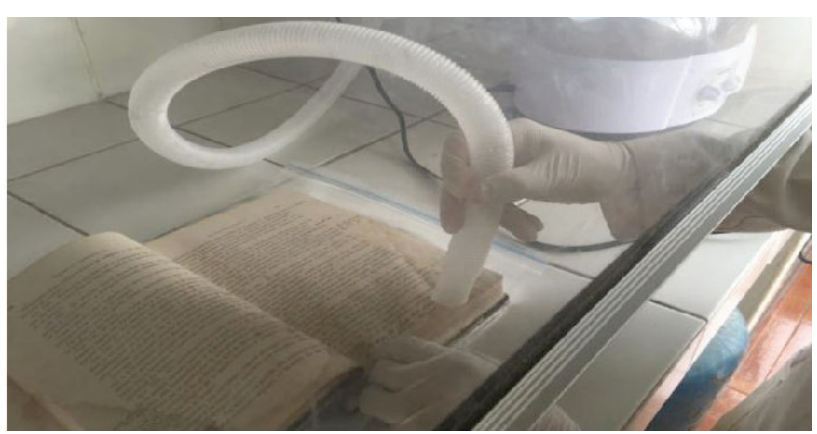

Figura 2. Aplicación de la solución B nebulizada sobre el libro

De esta forma se obtuvieron los siguientes datos (fig 3):

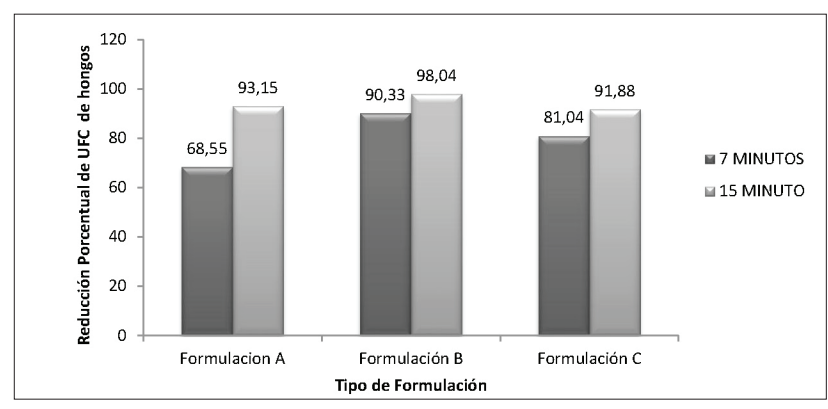

Figura 3. Comparación de la reducción porcentual de UFC de Hongos de las Formulaciones $A, B$ y $C$ a diferente tiempo de aplicación.

Como se puede observar en la Figura 3, la reducción porcentual de UFC de Hongos de la Formulación B $20 \%$ Agua: 30\% Etanol: 50\% Metanol), es mayor que la reducción porcentual de la Formulación A 1 $10 \%$ Agua: 50\% Etanol: 40\% Metanol) y C (10\% Agua: 60\% Etanol: $30 \%$ Metanol) a los dos tiempos de aplicación 7 y 15 minutos, esto quizás se deba a que la Formulación $B$ tiene más cantidad de agua con lo que la nebulización mejora y hay mayor contacto de la solución con el libro.

Las concentraciones de Fluconazol aumentan cuando existe mayor cantidad de metanol y Etanol (Formulación $A$ y C), pero disminuye el Nivel de Nebulización, esto se ve afectado en la reducción porcentual de UFC de Hongos de los mismos.

También se evaluó el efecto matriz de la mezcla de cosolventes, sin encontrar ningún efecto fungicida sobre los hongos aislados.

\section{Análisis estadístico de la Eficacia de las solu- ciones de Fluconazol}

\section{Optimización de la Respuesta}

Esta técnica ayuda a identificar los valores más probables de las variables que, en combinación, optimizaron una Reducción Porcentual de UFC del 100\%. Se tomaron las medias de cada repetición de los dos factores resumidos en la Tabla 5.

Tabla 5. Datos Experimentales para el diseño Factorial

\begin{tabular}{ccc}
\hline Factor 1 & Factor 2 (Tiempo de aplicación) \\
\hline Formulación A & $65,57 \%$ & 15 minutos \\
\hline & $65,18 \%$ & $92,69 \%$ \\
& $74,92 \%$ & $93,12 \%$ \\
\hline Formulación B & $90,66 \%$ & $93,69 \%$ \\
& $89,96 \%$ & $98,32 \%$ \\
& $90,37 \%$ & $98,24 \%$ \\
& $77,79 \%$ & $97,57 \%$ \\
\hline Formulación C & $81,38 \%$ & $92,01 \%$ \\
& $83,95 \%$ & $92,35 \%$ \\
& & $91,29 \%$ \\
\hline
\end{tabular}

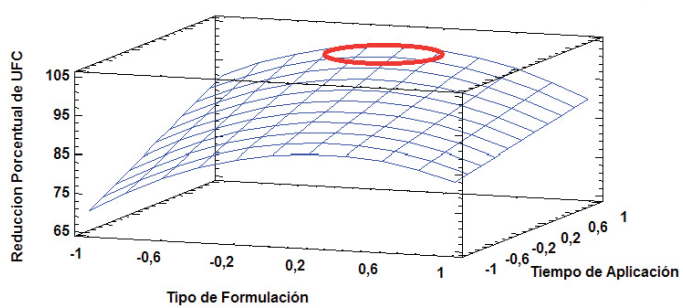

Figura 4. Optimización de Superficie de Respuesta del Tiempo de Aplicación y el Tipo de Formulación. 
El círculo que muestra la Figura 4 señala el área con mayor Reducción Porcentual estimada. Con valores que representan las Formulaciones $A, B, y C$ (valores de 1 a -1) y en Tiempo de Aplicación de 7 a 15 minutos, Obteniéndose así una Reducción Porcentual Estimada del $100 \%$ con la formulación B y 15 minutos para el tiempo de Aplicación.

\section{Conclusiones}

Se identificó mediante técnicas de aislamiento microbiológicas (muestreo de aire y muestreo de superficies por hisopado), dos familias principales de hongos presentes en los libros del Área Histórica: Aspergillus y, Penicillium.

Se determinó las proporciones adecuadas de la mezcla de solventes, mediante el diagrama pseudoternario, necesarias para obtener la mayor solubilidad de principio activo (Fluconazol), una nebulización aceptable, y una eficacia antifúngica adecuada para poder remplazar a los tratamientos comúnmente usados, encontrándose así que la proporción con la que se obtuvo Reducciones Porcentuales de UFC de Hongos mayores al 90\% fueron: Agua: 20\%: Metanol 50\%: Etanol $30 \%$.

Se determinó que existe un efecto estadísticamente significativo del tiempo de aplicación y del tipo de formulación concluyendo que a mayor tiempo de aplicación existe una mayor eficacia de las soluciones y que la composición de las mismas influye directamente en la eficacia de las soluciones.

Se identificó mediante la técnica de Optimización de respuesta que la mejor combinación para obtener Reducciones porcentuales de UFC de hongos de $100 \%$ es la Formulación B y el Tiempo de aplicación de 15 minutos.

\section{Referencias}

[1] SanMartín,N. (2015).Proyecto de Recuperación de la Memoria Historica y Conservación del Acervo, Quito: UCE.

[2] Aristegui, B. (2002). El reino de los hongos. Revista Iberoamericana de Micología, 2,3.

[3] Biasoli, M. (05 de 2013). Estructura y actividad de antifungicos. Recuperado el 20 de 07 de 2015, de http://www.fbioyf.unr.edu.ar/ evirtual/pluginfile.php/4130/course/section/1519/Estructura\%20y\%20actividad\%20de\%20los\%20antifungicos.pdf

[4] Selvaduray, G. (2004). Ternary phase diagrams.California.

[5] Farmacopea; Brazileña. (2010). Agencia Nacional de Vigilancia Sanitaria.Obtenido de http://www.anvisa.gov.br/hotsite/cd_farmacopeia/index.htm.

[6] Teran, R. (2015). Manual de Laboratorio de Micorbiologia Farmaceutica.Quito.

[7] Marcowist, B. (1990). How to prevent and remove mildew. Washington: Government Printing.

[8] Tangafire, V. (2011). UDEA. Obtenido de:

http://aprendeenlinea.udea.edu.co/lms/moodle/mod/resource/view.php?inpopup=true\&id=100812

[9] Avendaño, C. (2001). Introducción a la química farmacéutica (2a. ed.). Madrid, España: McGraw-Hill.

[10] USP32-NF27. (2009). The United States Pharmacopeial The National Formulary. (32a. ed.). 